\title{
Inteligencia emocional, optimismo y estrategias de afrontamiento en estudiantes universitarios
}

\section{Emotional intelligence, optimism and coping strategies in college students}

\author{
Laura Pardeiro, Josefa Castro \\ Universidad de Santiago de Compostela
}

\begin{abstract}
Resumen
Este trabajo explora la relación entre la inteligencia emocional, el optimismo, las estrategias de afrontamiento, y el rendimiento académico, en una muestra de 170 estudiantes universitarios. A los participantes se les aplicó el Schutte Self Report Emotional Inteligence Scale (SSREI), el Life Orientation Test (LOT-R), y el Coping Strategies Inventory (CSI). También se recogió la nota media del estudiante en el año anterior. Los resultados indican correlaciones positivas entre la inteligencia emocional, el optimismo, y el uso de estrategias de afrontamiento basadas en la resolución de problemas. No se encontraron relaciones estadísticamente significativas entre la inteligencia emocional y el rendimiento académico.

Palabras clave: inteligencia emocional, optimismo, estrategias de afrontamiento, logro académico
\end{abstract}

\begin{abstract}
This study examined the relationship between emotional intelligence, optimism, coping strategies and academic achievement, in a sample of 170 college students. Participants completed the Schutte Self Report Emotional Intelligence Scale (SSREI), the Life Orientation Test (LOT-R), and the Coping Strategies Inventory (CSI). Also were obtained students' last year academic final grades. Results showed positive correlations between emotional intelligence, optimism and use of coping strategies based on problem solving. No statistically significant relationships between emotional intelligence and academic performance were found.

Keywords: Emotional intelligence, optimism, coping strategies, academic achievement
\end{abstract}

La inteligencia emocional hace referencia a nuestra habilidad para reconocer, utilizar y comprender los estados emocionales propios y ajenos, con la finalidad de mejorar nuestros procesos cognitivos y facilitar nuestra adaptación al medio (Salovey y Mayer, 1990). En los últimos años la investigación entorno a la inteligencia emocional se ha enfocado en comprender como se relaciona este constructo con otras variables que influyen en el desarrollo del individuo.

Las investigaciones que han explorado la relación entre la inteligencia emocional y el rendimiento académico reflejan resultados diversos, debido en parte a la falta de acuerdo en cuanto a la conceptualización de la IE, y también a los instrumentos de medida que se emplean. En general, aquellos estudios que evalúan la IE con test de rendimiento tienden a obtener correlaciones significativas (Gil-Olarte, Palomera y Brackett, 2006; Rivers et al., 2012) no siendo tan concluyentes los resultados cuando los estudios emplean medidas de autoinforme (Petrides, Frederickson y Furnham, 2004; Mavrovelli y SanchezRuiz, 2011).

La inteligencia emocional también se ha vinculado con las estrategias de afrontamiento que utiliza un individuo para responder a las demandas externas o internas a las que se ve sometido. Los estudios indican el uso de tres tipos básicos de estrategias: las centradas en el problema, orientadas a modificar la demanda; las centradas en las emociones, orientadas a mitigar los sentimientos ocasionados por la demanda; y las enfocadas a la evitación del problema, y que buscan la evasión o negación del mismo. La inteligencia emocional parece influir en cómo afrontamos un evento estresante, minimizando o regulando las emociones negativas y potenciando las positivas (Downey, Johnston, Hansen, Birney y Stough, 2010; Mikolajczak, Nelis, Hansenne y Quoidbach, 2008; Petrides, Pérez-González y Furnham, 2007; Saklofske, Austin, Galloway y Davidson, 2007).

El optimismo también está relacionado con la utilización de estrategias de afrontamiento activas y focalizadas en la resolución del problema, frente a otras estrategias menos efectivas (negación o distanciamiento mental y conductual). Las personas optimistas, y que esperan resultados positivos, tenderían a emplear estrategias de afrontamiento efectivas y a ofrecer soluciones racionales. De ese modo, aumenta la probabilidad de éxito en la resolución de situaciones estresantes $y$, en consecuencia, el bienestar personal (Martínez, Reyes del Paso, García y González, 2006; Scheier y Carver, 1993).

Finalmente, la inteligencia emocional podría contribuir a la percepción optimista o pesimista que tenemos de los sucesos vitales cotidianos (Extremera, 
Durán y Rey, 2007).

A partir de los estudios previos, el objetivo de este trabajo es analizar la relación entre la inteligencia emocional, el optimismo, la estrategia de afrontamiento centrada en la resolución del problema y el rendimiento académico.

\section{Método}

\section{Participantes}

La muestra está formada por 170 estudiantes universitarios, 116 mujeres y 54 hombres, que cursaban el primer curso de grado en la Universidad de Santiago de Compostela. La edad media de los participantes fue de 19 años, con una desviación típica de 1,63, y un rango de edades entre 18 y 27 años.

\section{Instrumentos}

Los cuestionarios administrados en el estudio fueron los siguientes:

- Schutte Self Report Emotional Inteligence Scale (SSREI) de Schutte et al (1998), adaptada al español por Chico (1999). Se trata de una medida de autoinforme basado en el modelo conceptual de la IE de Salovey y Mayer (1990). Se compone de 33 ítems con un formato de respuesta tipo Likert de 5 puntos, que miden tres dimensiones: Valoración y expresión de las emociones, tanto en si mismo como en los demás, Regulación de las emociones, tanto en si mismo como en los demás y Utilización de las emociones de cara a resolver los problemas (planificación flexible, pensamiento creativo, atención y motivación dirigida).

- Test de orientación vital-Revisado (LOT-R), de Scheier, Carver y Bridges (1994), en la versión española de Otero, Luengo, Romero, Gómez-Fraguela y Castro (1998). Se compone de 10 ítems, medidos en escala Likert de 5 puntos ( $0=$ totalmente en desacuerdo, 4=totalmente de acuerdo), de los cuales 6 evalúan la dimensión de optimismo/pesimismo disposicional, y 4 actúan ocultando el contenido del test.

- Inventario de Estrategias de Afrontamiento (CSI), de Tobin, Holroyd y Reynolds (1994), en su versión española (Otero et al, 1998). El inventario completo consta de 40 ítems y mide ocho estrategias de afrontamiento diferentes. En el presente trabajo hemos utilizado solamente la subescala resolución de problemas, que incluye las estrategias cognitivas y conductuales dirigidas a cambiar la situación. Consta de 5 ítems que miden, en una escala Likert de 5 puntos ( $0=$ nunca utilizada, $4=$ siempre utilizada) el grado en el que la persona enfoca los esfuerzos de afrontamiento en la situación o problema.

- Para evaluar el Rendimiento Académico, se les pidió a los participantes que indicaran la nota media del Bachillerato.

\section{Procedimiento}

Los datos fueron recogidos en las aulas donde los alumnos asistían a las clases de sus materias del segundo cuatrimestre, en distintos centros de la universidad de Santiago de Compostela. Los cuestionarios se administraron siguiendo las normas de confidencialidad y consentimiento informado indicadas en el código ético.

\section{Análisis}

Los análisis se realizaron con el paquete estadístico IBM SPSS versión 20. En primer lugar, se llevó a cabo un análisis alfa de Cronbach para comprobar la fiabilidad de los instrumentos empleados. El análisis de la consistencia interna de las escalas mostró una fiabilidad adecuada, siendo mayor el alfa de Cronbach para la escala SSRI (.84) y la escala LOT-R (.80), y algo más baja para la subescala de Resolución de Problemas (.77). A continuación se llevó a cabo un análisis de correlación de Pearson para conocer si existía relación entre las puntuaciones de las distintas variables. Posteriormente, se efectuó un análisis de regresión múltiple "por pasos" para esclarecer cuales, de un conjunto de variables (valoración y expresión emocional, regulación emocional, utilización de las emociones, y resolución de problemas -criterio de entrada $\mathrm{F}<=.03-)$ son las que presentan una mayor capacidad predictiva del optimismo (variable criterio). Por último, se realizaron comparaciones de diferencias de medias mediante la prueba t de Student con el fin de analizar diferencias de género en la inteligencia emocional, optimismo y resolución de problemas.

\section{Resultados}

Las tres dimensiones que componen el constructo de inteligencia emocional, medidas con la escala SSREI, muestran correlaciones entre moderadas y altas con la puntuación global. El Factor que obtiene una mayor correlación con el constructo Inteligencia Emocional es el Factor 1 (Valoración y expresión de las emociones, tanto en sí mismo como en los otros) $(r=.86 ; \mathrm{p}<.001)$, seguido del Factor 2 (Regulación de las emociones, tanto en sí mismo como en los demás) $(r=.78 ; \mathrm{p}<.001)$ y del Factor 3 (Utilización de las emociones de cara a la resolución de problemas) $(r=.58 ; \mathrm{p}<.001)$.

Respecto a la relación entre la IE y el resto de variables, la Inteligencia Emocional correlaciona positiva y significativamente con el Optimismo $(r=.42$; $\mathrm{p}<.001)$. De los tres factores que componen la IE, el que correlaciona en mayor medida con el Optimismo es la Regulación emocional $(\mathrm{r}=.60 ; \mathrm{p}<.001)$ seguido de la Utilización de las emociones $(r=.30, p<.001)$.

La estrategia de resolución de problemas correlaciona de forma significativa, aunque con menor intensidad, tanto en la IE global $(\mathrm{r}=.40 ; \mathrm{p}<.001)$ como con sus tres dimensiones. El factor Regulación emocional vuelve a ser el de mayor índice de correlación ( $\mathrm{r}=.44 ; \mathrm{p}<.001)$, seguido del factor valoración/expresión emocional $(\mathrm{r}=.24 ; \mathrm{p}<.001)$ y utilización de las emociones $(\mathrm{r}=.20 ; \mathrm{p}<.001)$. También existe una correlación directa y significativa entre la resolución de problemas y el optimismo ( $\mathrm{r}=.39$; $\mathrm{p}<.001)$. El rendimiento académico no obtiene correlaciones significativas con ninguna de las variables del estudio.

A la vista de las correlaciones entre la IE, la resolución de problemas y el optimismo, y con la intención de explorar la capacidad predictiva de las 
mismas, efectuamos, como ya hemos avanzado, un análisis de regresión de pasos sucesivos, considerando como variable dependiente el optimismo y como predictores la resolución de problemas y los tres factores o dimensiones de la inteligencia emocional.

Tabla 1.

Intercorrelaciones entre Inteligencia Emocional, Optimismo, Resolución problemas y Rendimiento académico. Prueba de contraste: coeficiente correlación $r$ de Pearson

\begin{tabular}{|c|c|c|c|c|c|c|c|}
\hline Variable & RA & IEG & VEE & $\mathrm{RE}$ & $\mathrm{UE}$ & OPT & RP \\
\hline Rendimiento Académico (RA) & 1 & & & & & & \\
\hline Intel. Emoc. Global (IEG) & .06 & 1 & & & & & \\
\hline Valoración/Expresión emoc (VEE) & .03 & $.87^{* *}$ & 1 & & & & \\
\hline Regulación emocional (RE) & .07 & $.78^{*}$ & $.42^{* *}$ & 1 & & & \\
\hline Utilización emociones (UE) & .06 & $.58^{* *}$ & $.30^{*}$ & $.30^{*}$ & 1 & & \\
\hline Optimismo (OPT) & .04 & $.42^{* *}$ & .14 & $.60^{\text {** }}$ & $.30^{* *}$ & 1 & \\
\hline Resolución problemas (RP) & .06 & $.40^{* *}$ & $.24^{* *}$ & $.44^{* *}$ & $.20^{*}$ & $.39^{* *}$ & 1 \\
\hline
\end{tabular}

$\mathrm{N}=170$ * $\mathrm{p}<.01 . * * \mathrm{p}<.001$

Tabla 2.

Análisis de regresión múltiple por pasos, tomando como VD el optimismo, y como VI un conjunto de variables (valoración-expresión emocional, regulación emocional, utilización de las emociones, resolución de problemas).

\begin{tabular}{ccccccc}
\hline Pasos & Variables & $F$ & Sig & $R$ & $R^{2}$ corregida & ${\text { Cambio en } R^{2}}^{2}$ \\
\hline 1 & Regulación emocional & 90.448 & .001 & .592 & .346 & .350 \\
2 & Resolución de problemas & 48.922 & .001 & .608 & .362 & .019 \\
\hline
\end{tabular}

El análisis de regresión muestra que el principal predictor es la variable regulación emocional que contribuye con un $34,6 \%$ a la variable criterio, y como segundo paso del análisis es seleccionada la variable resolución de problemas que contribuye en un $1,9 \%$ a la explicación del optimismo. En definitiva, las dos variables contribuyen en un $36,2 \%$ al optimismo.

Finalmente, se ha analizado si existían diferencias de género. La puntuación media en Inteligencia emocional en nuestra muestra es de 120,1 sobre un máximo de 165, situándola así en una posición ligeramente más baja que la encontrada en el estudio de Chico (1999), en el cual también medía dicho constructo en una muestra de estudiantes $(M=126)$. La puntuación media obtenida en la escala de Optimismo, es de 14.7, muy similar a los datos normativos del test en una muestra de estudiantes de universidad (puntuación media de 14.33). En cuanto a la puntuación obtenida en la subescala de Resolución de Problemas, es de $M=12.82$, en un máximo de 20. Las mujeres tienen una puntuación media global más alta en la escala de IE que los hombres $(M=120.84, D T=11.96$ y $M=118.52$, $D T=12.52$ respectivamente), pero no es significativa estadísticamente $(\mathrm{t}=1.147, \mathrm{p}=.25)$. Las puntuaciones son muy similares en la regulación emocional (mujeres $M=39.33, D T=5.54$; hombres $M=39.96, D T=5.17$ ) y en la utilización de las emociones (mujeres $M=27.27$, $D T=3.04$ y hombres $M=26.67, D T=3.81$ ). En cambio, hay diferencias significativas en la valoración y expresión emocional, factor en el que las mujeres puntúan más alto que los hombres (mujeres $M=54,21$, $D T=6.86$ y hombres $M=51.89, D T=7.74)\left(\mathrm{t}_{168}=1.996\right.$, $p<.05)$. No se aprecian diferencias significativas entre hombres y mujeres en las puntuaciones en la escala de optimismo ni en la utilización de la estrategia de resolución de problemas.

\section{Discusión}

El propósito de este estudio ha sido explorar la relación entre el constructo inteligencia emocional, el optimismo, la resolución de problemas y el rendimiento académico.

Nuestros resultados no muestran relación entre el logro académico y la inteligencia emocional global ni con ninguno de los factores que componen dicho constructo. Si bien estos datos concuerdan con los de otros estudios que tampoco obtienen una relación entre estas dos variables (e.g. Hansenne y Legrand, 2012; Jiménez y López-Zafra, 2013; Mavroveli y SánchezRuiz, 2011), creemos que también podría deberse a una limitación de nuestro estudio, al emplear como medida del rendimiento académico la media del bachillerato. Medida que ha limitado la variabilidad entre los sujetos.

Respecto al resto de variables analizadas, los datos indican una relación moderada y positiva entre la inteligencia emocional, la resolución de problemas y el optimismo.

En relación a las estrategias de afrontamiento, niveles altos en inteligencia emocional se relacionan con estrategias orientadas a buscar soluciones lógicas a los problemas. Concretamente, la regulación emocional y la utilización de las emociones, son las dimensiones que tienen una mayor relación con la resolución de problemas. Estos datos concuerdan con otros estudios que también resaltan el papel de la autorregulación y la gestión adecuada de las emociones como una capacidad que facilitaría el uso de estrategias más adaptativas (Austin, Saklofske y Mastoras, 2010; Martínez, Piqueras e Inglés, 2011). 
Tabla 3.

Medias, desviaciones típicas y diferencias de género en las variables del estudio (rendimiento académico, inteligencia emocional, optimismo y resolución de problemas).

\begin{tabular}{lccccc}
\hline \multicolumn{1}{c}{ Variables (rango) } & Total & Mujeres & Hombres & $t$ & $p$ \\
& $\mathrm{~N}=170$ & $\mathrm{~N}=116$ & $\mathrm{~N}=54$ & $(\mathrm{gl}=168)$ \\
& $M(D T)$ & $M(D T)$ & $M(D T)$ & & .381 \\
\hline Rendimiento académico & $7.6(0.93)$ & $7.62(0.94)$ & $7.56(0.91)$ & .704 \\
IE Global (33-165) & $120.1(12.27)$ & $120.84(11.96)$ & $118.52(12.88)$ & 1.147 & .253 \\
Expresión emocional (15-75) & $53.49(7.2)$ & $54.24(6.86)$ & $51.89(7.74)$ & 1.996 & .048 \\
Regulación emocional (11-55) & $39.53(5.4)$ & $39.33(5.54)$ & $39.96(5.17)$ & -.710 & .635 \\
Utilización emociones (7-35) & $27.08(3.3)$ & $27.27(3.04)$ & $26.67(3,81)$ & 1.104 & .271 \\
Optimismo (0-24) & $14.7(5.38)$ & $14.77(5.25)$ & $14.55(5.69)$ & .238 & .812 \\
Resolución problemas (0-20) & $12.82(3.29)$ & $12.71(3.26)$ & $13.06(3.26)$ & -.648 & .518 \\
\hline
\end{tabular}

Prueba constraste: Prueba t de student para dos muestras independientes

Nuestros resultados $(\mathrm{r}=.40 ; \mathrm{p}<.001)$ también apoyan la vinculación entre estrategias de afrontamiento y optimismo descrita en la literatura. Las personas optimistas tienen un estilo de afrontamiento al estrés basado en el empleo de estrategias activas, mientras que los pesimistas emplean estrategias de afrontamiento pasivo. Esta relación explicaría porque las personas con pensamientos optimistas manifiestan un mayor bienestar psicológico (Scheier, Weintraub y Carver, 1986; Carver y Scheier, 2001).

Los estudios que exploran la relación entre la inteligencia emocional y el optimismo disposicional medido a través del LOT-R constatan correlaciones moderadas entre ambas variables (Augusto-Landa, Pulido-Martos y López-Zafra, 2011; Extremera et al, 2007; Brown y Schutte, 2006). Los resultados encontrados en este trabajo van en la línea de los propuestos por los autores anteriores. El constructo Inteligencia emocional y el optimismo correlacionaron positiva y significativamente $(r=.42 ; \mathrm{p}<.01)$, siendo la habilidad de regulación emocional la que mostró una correlación más elevada $(r=.60 ; \mathrm{p}<.01)$.

Finalmente, el análisis de regresión permite constatar que es la regulación emocional, frente a las restantes variables (valoración-expresión emocional, utilización emocional), aquella que presenta la mayor capacidad predictiva $(35 \%)$ del optimismo. La segunda variable que conforma la ecuación es la resolución de problemas. Las dos variables contribuyen en un $36 \%$ al optimismo. A raíz de nuestros resultados, consideramos que los programas de intervención dirigidos a incrementar la IE de los estudiantes, deberían incidir en mejorar las destrezas de autorregulación emocional con el fin de promover el optimismo y aumentar así el bienestar personal.

Por último, y en concordancia con los resultados de otros estudios (Aritzea, Balluerka, Barberá y Gartzia, 2012; Tsaousis y Kazi, 2013) las mujeres obtienen puntuaciones significativamente más altas en inteligencia emocional $(\mathrm{p}<.05)$, especialmente en lo que concierne a la valoración y expresión de las emociones.

\section{Referencias}

Aritzeta, A., Balluerka, N., Barberá, E. y Gartzia, L. (2012). Inteligencia emocional y género: más allá de las diferencias sexuales. Anales de Psicología, 28 (2), 567-575.

Augusto-Landa, J. M., Pulido-Martos, M. y LopezZafra, E. (2011). Does perceived emotional intelligence and optimism/pessimism predict psychological well-being?. Journal of Happiness Studies, 12(3), 463-474.

Austin, E. J., Saklofske, D. H. y Mastoras, S. M., (2010). Emotional intelligence, coping and examrelated stress in Canadian undergraduate students. Australian Journal of Psychology, 62 (1), 42-50.

Brown, R. F. y Schutte, N. S. (2006). Direct and indirect relationships between emotional intelligence and subjective fatigue in university students. Journal of Psychosomatic Research, 60(6), 585-593.

Carver, C. S. y Scheier, M. F. (2001). Optimism, pessimism, and self-regulation. In E. C. Chang (Ed.), Optimism and pessimism: Implications for theory, research, and practice, 31-51. Washington, DC: American Psychological Association.

Chico, E. (1999). Evaluación Psicométrica de una Escala de Inteligencia Emocional. Boletín de Psicología ,Vol 62, 65-78.

Downey, L.A., Johnston, P.J., Hansen, K., Birney, J., y Stough, C. (2010). Investigating the mediating effects of emotional intelligence and coping on problem behaviours in adolescents. Australian Journal of Psychology, 62 (1), 20-29.

Extremera, N., Durán, A. y Rey, L. (2007). Perceived emotional intelligence and dispositional optimismpessimism: Analyzing their role in predicting psychological adjustment among adolescents. Personality and Individual Differences, 42 (6), 10691079.

Gil-Olarte, P., Palomera, R. y Brackett, M. A. (2006). Relating emotional intelligence to social competence and academic achievement in high school students. Psicothema, 18, 118-123.

Hansenne, M. \& Legrand, J. (2012). Creativity, emotional intelligence, and school performance in 
children. International Journal of Educational Research, 53, 264-268.

Jiménez, M. I. y López-Zafra, E. (2013). Impacto de la Inteligencia Emocional Percibida, Actitudes Sociales y Expectativas del Profesor en el Rendimiento Académico. Electronic Journal of Research in Educational Psychology, 11(29), 75-98.

Martínez, A., Reyes del Paso, G., García, A. y González, I. (2006). Optimismo/ pesimismo disposicional y estrategias de afrontamiento del estrés. Psicothema, 18 (1), 66-72.

Martínez, A.E., Piqueras, J.A. e Inglés, C. J. (2011). Relaciones entre inteligencia emocional y estrategias de afrontamiento ante el estrés. Revista electrónica de motivación y emoción, 37, 20-21.

Mavroveli, S. y Sánchez-Ruiz, M.J. (2011). Trait emotional intelligence influences on academic achievement and school behaviour. British Journal of Educational Psychology, 81(1), 112-134.

Mikolajczak, M., Nelis, D., Hansenne, M. y Quoidbach, J. (2008). If you can regulate sadness, you can probably regulate shame: Associations between trait emotional intelligence, emotion regulation and coping efficiency across discrete emotions. Personality and Individual Differences, 44 (6), 1356-1368.

Otero, J. M., Luengo, A., Romero, E., GómezFraguela, J. A. y Castro, C. (1998). Psicología de la Personalidad. Manual de practicas. Barcelona: Ariel.

Petrides, K. V. y Furnham, A. (2001). Trait Emotional Intelligence: Psychometric Investigation with Reference to Established Trait Taxonomies. European Journal of Personality, 15 (6), 425-448.

Petrides, K. V., Frederickson, N. y Furnham, A. (2004). The role of trait emotional intelligence in academic performance and deviant behavior at school. Personality and Individual Differences, 36, 277-293.

Petrides, K. V., Pérez-González, J. C. y Furnham, A. (2007). On the criterion and incremental validity of trait emotional intelligence. Cognition \& Emotion, 21, 26-55.

Qualter, P., Gardner, K., Pope, D., Hutchinson, J. \& Whiteley, H. (2012). Ability emotional intelligence, trait emotional intelligence, and academic success in British secondary schools: a 5 year longitudinal study. Learning and Individual Differences, 22, 8391.

Rivers, S. E., Brackett, M. A., Reyes, M. R., Mayer, J. D., Caruso, D. y Salovey, P. (2012). Measuring emotional intelligence in early adolescence with the MSCEIT-YV: Psychometric properties and relationship with academic performance and psychosocial functioning. Journal of Psychoeducational Assessment, 30 (4), 344- 366.

Saklofske, D. H., Austin, E. J., Galloway, J. y Davidson, K. (2007). Individual difference correlates of health-related behaviours: Preliminary evidence for links between emotional intelligence and coping. Personality and Individual Differences, 42, 491-502.
Salovey, P. y Mayer, J. D. (1990). Emotional intelligence. Imagination, Cognition, and Personality, 9 (3), 185-211.

Scheier, M.F.; Weintraub, J.K. y Carver, C.S. (1986). Coping with stress: Divergent strategies of optimists and pessimists. Journal of Personality and Social Psychology, 51, 1257-1264.

Scheier, M. F. y Carver, C. S. (1993). On the Power of Positive Thinking: The Benefits of Being Optimistic. Current Directions in Psychological Science, 2 (1), 26-30.

Scheier, M.F., Carver, C.S. y Bridges, M.W. (1994). Distinguishing optimism from neuroticism (and trait anxiety, self-mastery, and self-esteem): a reevaluation of the life orientation test. Journal of Personality and Social Psychology, 67 (6), 10631078.

Schutte, N. S., Malouff, J. M., Hall, L., Haggerty, D., Cooper, J., Golden, C. y Dornheim, L. (1998). Development and validation of a measure of emotional intelligence. Personality and Individual Differences, 25 (2), 167-177.

Tobin, D.J., Holroyd, K.A. y Reynolds, R.V.C. (1984). Users manual for Coping Strategies Inventory. Ohio University Press.

Tsaousis, I. y Kazi, S. (2013). Factorial invariance and latent mean differences of scores on trait emotional intelligence across gender and age. Personaliy and Individual Differences, 54(2), 169-173. 